\title{
A Generalized Higher Reverse Left (respectively Right) Centralizer on Prime $\Gamma$-Rings
}

\author{
Fawaz Ra'ad Jarullah Salah Mehdi Salih \\ Department of Mathematics , College of Education , Al-Mustansirya University , Iraq
}

\begin{abstract}
:
This study introduces the concepts of generalized higher reverse left (respectively right) centralizer, Jordan generalized higher reverse left (respectively right) centralizer and Jordan triple generalized higher reverse left (respectively right) centralizer of Gamma-rings. In this paper we prove the following main results . Every Jordan generalized higher reverse left (respectively right) centralizer of a 2-torsion free prime $\Gamma$-ring $\mathrm{M}$ into itself is generalized higher reverse left (respectively right) centralizer of $\mathrm{M}$ and every Jordan generalized higher reverse left (respectively right ) centralizer of a 2-torsion free $\Gamma$-ring $\mathrm{M}$, into itself, such that $\mathrm{x} \alpha \mathrm{y} \beta \mathrm{x}=\mathrm{x} \beta \mathrm{y}$ $\alpha \mathrm{x}$ is a Jordan triple generalized higher reverse left( respectively right ) centralizer of $\mathrm{M}$, for all $\mathrm{x}, \mathrm{y} \in \mathrm{M}$ and $\alpha, \beta \in \Gamma$
\end{abstract}

It is noteworthy that $\mathbf{T}_{\mathrm{o}}(\mathbf{x})=\mathbf{x}$ and $\mathbf{T}_{\mathrm{o}}(\mathbf{x} \alpha \mathbf{y})=\mathbf{y} \alpha \mathbf{x}$, for all $a, b \in \mathbf{M}$ and $\alpha \in \Gamma$.

Key Words : prime $\Gamma$-ring, left centralizer, right centralizer, Jordan centralizer

Mathematic Subject classification : 16N60, 16W25, 16Y99

DOI: $10.7176 / \mathrm{MTM} / 9-10-06$

Publication date: October $31^{\text {st }} 2019$

\section{1-INTRODUCTION:}

Let $M$ and $\Gamma$ be two additive a belian groups. Suppose that there is a mapping from $M \times \Gamma \times M \longrightarrow M$ (the image of $(\mathrm{x}, \alpha, \mathrm{y})$ denoted by $\mathrm{x} \alpha \mathrm{y}$, where $\mathrm{x}, \mathrm{y} \in \mathrm{M} \quad$ and $\alpha \in \Gamma$ ) satisfying the following properties for all $\mathrm{x}, \mathrm{y}, \mathrm{z} \in \mathrm{M}$ and $\alpha, \beta \in \Gamma$

(i) $\quad(\mathrm{x}+\mathrm{y}) \alpha \mathrm{z}=\mathrm{x} \alpha \mathrm{z}+\mathrm{y} \alpha \mathrm{z}$

$$
\begin{aligned}
& \mathrm{x}(\alpha+\beta) \mathrm{z}=\mathrm{x} \alpha \mathrm{z}+\mathrm{x} \beta \mathrm{z} \\
& \mathrm{x} \alpha(\mathrm{y}+c)=\mathrm{x} \alpha \mathrm{y}+\mathrm{x} \alpha \mathrm{z}
\end{aligned}
$$

(ii) $\quad(\mathrm{x} \alpha \mathrm{y}) \beta \mathrm{z}=\mathrm{x} \alpha(\mathrm{y} \beta \mathrm{z})$.

Then $\mathrm{M}$ is called a $\Gamma$-ring. [ 1 ].

$\mathrm{M}$ is called a prime if $\mathrm{x} \Gamma \mathrm{M} \Gamma \mathrm{y}=(0)$ implies that $\mathrm{x}=0$ or $\mathrm{y}=0$, where $\mathrm{x}, \mathrm{y} \in \mathrm{M}[5]$ ].

$\mathrm{M}$ is called a semiprime if $\mathrm{x} \Gamma \mathrm{M} \Gamma \mathrm{x}=(0)$ implies that $\mathrm{x}=0$, where $\mathrm{x} \in \mathrm{M} \quad[5]$.

$\mathrm{M}$ is called a 2-torsion free if $2 \mathrm{x}=0$ implies that $\mathrm{x}=0$, for all $\mathrm{x} \in \mathrm{M}$ [5] .

If $\mathrm{M}$ is a $\Gamma$-ring, then $[\mathrm{x}, \mathrm{y}]_{\alpha}=\mathrm{x} \alpha \mathrm{y}-\mathrm{y} \alpha \mathrm{x}$, for all $\mathrm{x}, \mathrm{y} \in \mathrm{M}$ and $\alpha \in \Gamma$,is known as a commutator . [5 ]

An additive mapping $\mathrm{d}: \mathrm{M} \longrightarrow \mathrm{M}$ is called a derivation if the following holds :

$\mathrm{d}(\mathrm{x} \alpha \mathrm{y})=\mathrm{d}(\mathrm{x}) \alpha \mathrm{y}+\mathrm{x} \alpha \mathrm{d}(\mathrm{y})$, for all $\mathrm{x}, \mathrm{y} \in \mathrm{M}$ and $\alpha \in \Gamma[2]$ 
Additionally, $\mathrm{d}$ is called a Jordan derivation if the following property holds :

$\mathrm{d}(\mathrm{x} \alpha \mathrm{x})=\mathrm{d}(\mathrm{x}) \alpha \mathrm{x}+\mathrm{x} \alpha \mathrm{d}(\mathrm{x})$, for all $\mathrm{x} \in \mathrm{M}$ and $\alpha \in \Gamma$. [2]

An additive mapping $\mathrm{F}: \mathrm{M} \longrightarrow \mathrm{M}$ is called a generalized derivation associated with the derivation $\mathrm{d}: \mathrm{M}$

$\longrightarrow \mathrm{M}$ if the following equation holds :

$\mathrm{F}(\mathrm{x} \alpha \mathrm{y})=\mathrm{F}(\mathrm{x}) \alpha \mathrm{y}+\mathrm{x} \alpha \mathrm{d}(\mathrm{y})$, for all $\mathrm{x}, \mathrm{y} \in \mathrm{M}$ and $\alpha \in \Gamma[2]$

In addition, $\mathrm{F}$ is called a Jordan generalized derivation associated with the Jordan derivation $\mathrm{d}: \mathrm{M} \longrightarrow \mathrm{M}$ if the following property is satisfied :

$\mathrm{F}(\mathrm{x} \alpha \mathrm{x})=\mathrm{F}(\mathrm{x}) \alpha \mathrm{x}+\mathrm{x} \alpha \mathrm{d}(\mathrm{x})$, for all $\mathrm{x} \in \mathrm{M}$ and $\alpha \in \Gamma[2]$

A left (respectively right) centralizer of a $\Gamma$-ring $\mathrm{M}$, is an additive mapping, $\mathrm{T}: \mathrm{M} \longrightarrow \mathrm{M}$ which satisfies the following equation $\mathrm{T}(\mathrm{x} \alpha \mathrm{y})=\mathrm{T}(\mathrm{x}) \alpha \mathrm{y}$ (respectively $\mathrm{T}(\mathrm{x} \alpha \mathrm{y})=\mathrm{x} \alpha \mathrm{T}(\mathrm{y}))$, for all $\mathrm{x}, \mathrm{y} \in \mathrm{M}$ and $\alpha \in \Gamma \mathrm{T}$ is called a centralizer of $\mathrm{M}$ if it is both a left and right centralizer [ 4]. A left (respectively right) Jordan centralizer of a $\Gamma$-ring $M$, is an additive mapping, $\mathrm{T}: \mathrm{M} \longrightarrow \mathrm{M}$ which satisfies the following equation $\mathrm{T}(\mathrm{x} \alpha \mathrm{x})=\mathrm{T}(\mathrm{x}) \alpha \mathrm{x}$ (respectively $\mathrm{T}(\mathrm{x} \alpha \mathrm{x})=\mathrm{x} \alpha \mathrm{T}(\mathrm{x}))$, for all $\mathrm{x} \in \mathrm{M}$ and $\alpha \in$ Г. $\mathrm{T}$ is called a Jordan centralizer of $\mathrm{M}$ if it is both a left and right Jordan centralizer [ 4 ] .

An additive mapping $\mathrm{F}: \mathrm{M} \longrightarrow \mathrm{M}$ is called a generalized centralizer of $\mathrm{M}$ associated with the centralizer $\mathrm{T}: \mathrm{M} \longrightarrow \mathrm{M}$ if the following equation holds :

$\mathrm{F}(\mathrm{x} \alpha \mathrm{y}+\mathrm{y} \beta \mathrm{x})=\mathrm{F}(\mathrm{x}) \alpha \mathrm{y}+\mathrm{y} \beta \mathrm{T}(\mathrm{x})$, for all $\mathrm{x}, \mathrm{y} \in \mathrm{M}$ and $\alpha, \beta \in \Gamma[3]$.

$\mathrm{F}$ is called a Jordan generalized centralizer of $\mathrm{M}$ associated with the Jordan centralizer $\mathrm{T}: \mathrm{M} \longrightarrow$

$\mathrm{M}$ if the following equation holds :

$\mathrm{F}(\mathrm{x} \alpha \mathrm{x}+\mathrm{x} \alpha \mathrm{x})=\mathrm{F}(\mathrm{x}) \alpha \mathrm{x}+\mathrm{x} \alpha \mathrm{T}(\mathrm{x})$, for all $\mathrm{x} \in \mathrm{M}$ and $\alpha, \beta \in \Gamma$ [3].

Let $T=\left(t_{i}\right)_{i \in N}$ be a family of additive mappings of a ring $R$, into itself . Then $T$ is called a higher left centralizer, we have that

$\mathbf{t}_{\mathrm{n}}(\mathrm{xy})=\sum_{\mathrm{i}=1}^{\mathrm{n}} \mathbf{t}_{\mathrm{i}}(\mathrm{x}) \mathrm{t}_{\mathrm{i}-1}(\mathrm{y})$, for all $\mathrm{x}, \mathrm{y} \in \mathrm{R}$ and $\mathrm{n} \in \mathrm{N}$ [6].

In addition, $\mathrm{T}$ is called a Jordan higher left centralizer if the following equation holds :

$\mathbf{t}_{\mathrm{n}}\left(\mathrm{x}^{2}\right)=\sum_{\mathrm{i}=1}^{\mathrm{n}} \mathrm{t}_{\mathrm{i}}(\mathrm{x}) \mathrm{t}_{\mathrm{i}-1}(\mathrm{x})$, for all $\mathrm{x} \in \mathrm{R}$ and $\mathrm{n} \in \mathrm{N}[6]$.

Let $\mathrm{F}=\left(f_{\mathrm{i}}\right)_{\mathrm{i} \in \mathrm{N}}$ be a family of additive mappings of a ring $\mathrm{R}$, into itself .Then $\mathrm{F}$ is called a generalized higher left centralizer associated with the higher left centralizer $T=\left(t_{i}\right) i \in N$ of $R$, if the following equation is satisfied : $f_{\mathrm{n}}(\mathrm{xy})=\sum_{\mathrm{i}=1}^{\mathrm{n}} f_{\mathrm{i}}(\mathrm{x}) \mathrm{t}_{\mathrm{i}-1}(\mathrm{y})$, for all $\mathrm{x}, \mathrm{y} \in \mathrm{R}$ and $\mathrm{n} \in \mathrm{N}[6]$

Moreover, F is called a Jordan generalized higher left centralizer associated with the Jordan higher left centralizer $T=\left(t_{i}\right)_{i \in N}$ of $R$, we have that $f_{\mathrm{n}}\left(\mathrm{x}^{2}\right)=\sum_{\mathrm{i}=1}^{\mathrm{n}} f_{\mathrm{i}}(\mathrm{x}) \mathrm{t}_{\mathrm{i}-1}(\mathrm{x})$, for all $\mathrm{x} \in \mathrm{R}$ and $\mathrm{n} \in \mathrm{N}[6]$.

Jarullah and Salih introduced the concepts of higher reverse left (resp. right) centralizer and Jordan higher reverse left ( respectively right ) centralizer a $\Gamma$-ring as follows : 
Let $t=\left(t_{i}\right)_{i \in N}$ be a family of additive mappings of a $\Gamma$-ring $M$ into itself. Then $t$ is called a higher reverse left ( respectively right ) centralizer of $\mathrm{M}$, we have that

$t_{n}(x \propto y)=\sum_{i=1}^{n} t_{i}(y) \alpha t_{i-1}(x)$

$\left(\right.$ respectively $\left.\mathbf{t}_{\mathrm{n}}(\mathrm{x} \alpha \mathrm{y})=\sum_{\mathrm{i}=1}^{\mathrm{n}} \mathrm{t}_{\mathrm{i}-1}(\mathrm{y}) \alpha \mathbf{t}_{\mathrm{i}}(\mathrm{x})\right)$, for all $\mathrm{x}, \mathrm{y} \in \mathrm{M}, \alpha \in \Gamma$ and $\mathrm{n} \in \mathrm{N}$ [7]

Let $t=\left(t_{i}\right)_{i \in N}$ be a family of additive mappings of a $\Gamma$-ring $M$ into itself .Then $t$ is called a Jordan higher reverse left (respectively right) centralizer of $\mathrm{M}$ if the following equation holds :

$\mathrm{t}_{\mathrm{n}}(\mathrm{x} \alpha \mathrm{x})=\sum_{\mathrm{i}=1}^{\mathrm{n}} \mathrm{t}_{\mathrm{i}}(\mathrm{x}) \alpha \mathrm{t}_{\mathrm{i}-1}(\mathrm{x})$

(respectively $\left.\mathrm{t}_{\mathrm{n}}(\mathrm{x} \alpha \mathrm{x})=\sum_{\mathrm{i}=1}^{\mathrm{n}} \mathrm{t}_{\mathrm{i}-1}(\mathrm{x}) \alpha \mathrm{t}_{\mathrm{i}}(\mathrm{x})\right)$, for all $\mathrm{x} \in \mathrm{M}, \alpha \in \Gamma$

and

$\mathrm{n} \in \mathrm{N}[7]$

Jarullah and Salih introduced the concepts of higher reverse left (respectively right) centralizer, Jordan higher reverse left ( respectively right) centralizer and generalization on Rings as follows :

Let $t=\left(t_{i}\right)_{i \in N}$ be a family of additive mappings of a ring $R$ into itself . Then $t$ is called a higher reverse left ( respectively right) centralizer of $\mathrm{R}$, we have that

$\mathbf{t}_{\mathrm{n}}(\mathrm{xy})=\sum_{\mathrm{i}=1}^{\mathrm{n}} \mathrm{t}_{\mathrm{i}}(\mathrm{y}) \mathrm{t}_{\mathrm{i}-1}(\mathrm{x})$

( respectively $\left.\mathbf{t}_{\mathrm{n}}(\mathrm{xy})=\sum_{\mathrm{i}=1}^{\mathrm{n}} \mathrm{t}_{\mathrm{i}-1}(\mathrm{y}) \mathrm{t}_{\mathrm{i}}(\mathrm{x})\right)$, for all $\mathrm{x}, \mathrm{y} \in \mathrm{R}$ and $\mathrm{n} \in \mathrm{N}$ [8] .

Let $\mathrm{t}=\left(\mathrm{t}_{\mathrm{i}}\right)_{\mathrm{i} \in \mathrm{N}}$ be a family of additive mappings of a ring $\mathrm{R}$ into itself . Then $\mathrm{t}$ is called a Jordan higher reverse left (respectively right) centralizer of $\mathrm{R}$, if the following equation holds :

$t_{n}\left(x^{2}\right)=\sum_{i=1}^{n} t_{i}(x) t_{i-1}(x)$

(respectively $\left.\mathbf{t}_{\mathrm{n}}\left(\mathrm{x}^{2}\right)=\sum_{\mathrm{i}=1}^{\mathrm{n}} \mathrm{t}_{\mathrm{i}-1}(\mathrm{x}) \mathrm{t}_{\mathrm{i}}(\mathrm{x})\right)$, for all $\mathrm{x} \in \mathrm{R}$ and $\mathrm{n} \in \mathrm{N} \quad[8]$.

Let $\mathrm{T}=\left(\mathrm{T}_{\mathrm{i}}\right)_{\mathrm{i} \in \mathrm{N}}$ be a family of additive mappings of a ring $\mathrm{R}$ into itself .Then $\mathrm{T}$ is called

a generalized higher reverse left ( respectively right) centralizer of a ring $\mathrm{R}$ into itself associated with the higher reverse left ( respectively right) centralizer $t=\left(t_{i}\right)_{i \in N}$ of $R, \quad$ such that

$\mathrm{T}_{\mathrm{n}}(\mathrm{xy})=\sum_{\mathrm{i}=1}^{\mathrm{n}} \mathrm{T}_{\mathrm{i}}(\mathrm{y}) \mathrm{t}_{\mathrm{i}-1}(\mathrm{x})$

( respectively $\left.\mathbf{T}_{\mathrm{n}}(\mathrm{xy})=\sum_{\mathrm{i}=1}^{\mathrm{n}} \mathbf{t}_{\mathrm{i}-1}(\mathrm{y}) \mathbf{T}_{\mathrm{i}}(\mathbf{x})\right)$, for all $\mathrm{x}, \mathrm{y} \in \mathrm{R}$ and $\mathrm{n} \in \mathrm{N}$ [9] 
Let $\mathrm{T}=\left(\mathrm{T}_{\mathrm{i}}\right)_{\mathrm{i} \in \mathrm{N}}$ be a family of additive mappings of a ring $\mathrm{R}$ into itself . Then $\mathrm{T}$ is called a generalized Jordan higher reverse left ( respectively right) centralizer of a ring $\mathrm{R}$ into itself associated with Jordan higher reverse left ( respectively right) centralizer $\mathrm{t}=\left(\mathrm{t}_{\mathrm{i}}\right)_{\mathrm{i} \in \mathrm{N}}$ of $\mathrm{R}$, such that

$\mathrm{T}_{\mathrm{n}}\left(\mathrm{x}^{2}\right)=\sum_{\mathrm{i}=1}^{\mathrm{n}} \mathrm{T}_{\mathrm{i}}(\mathrm{x}) \mathrm{t}_{\mathrm{i}-1}(\mathrm{x})$

( respectively $\left.\mathbf{T}_{\mathrm{n}}\left(\mathrm{x}^{2}\right)=\sum_{\mathrm{i}=1}^{\mathrm{n}} \mathbf{t}_{\mathrm{i}-1}(\mathrm{x}) \mathbf{T}_{\mathrm{i}}(\mathbf{x})\right)$, for all $\mathrm{x} \in \mathrm{R}$ and $\mathrm{n} \in \mathrm{N}$ [9]

\section{2- Generalized Higher Reverse Left (Respectively Right) Centralizer on Prime $\Gamma$-Rings}

\section{Definition ( 2.1 )}

Let $\mathrm{T}=\left(\mathrm{T}_{\mathrm{i}}\right)_{\mathrm{i} \in \mathrm{N}}$ be a family of additive mappings of a $\Gamma$-ring $\mathrm{M}$ into itself. Then $\mathrm{T}$ is called a generalized higher reverse left (respectively right) centralizer of the $\Gamma$-ring $M$, associated with the higher reverse left (respectively right) centralizer $\mathrm{t}=\left(\mathrm{t}_{\mathrm{i}}\right)_{\mathrm{i} \in \mathrm{N}}$ of the $\quad \Gamma$-ring $\mathrm{M}$, into itself if, for all $\mathrm{x}, \mathrm{y} \in \mathrm{M}, \alpha \in \Gamma$ and $\mathrm{n} \in \mathrm{N}$

$\mathrm{T}_{\mathrm{n}}(\mathrm{x} \alpha \mathrm{y})=\sum_{\mathrm{i}=1}^{\mathrm{n}} \mathrm{T}_{\mathrm{i}}(\mathrm{y}) \alpha \mathrm{t}_{\mathrm{i}-1}(\mathrm{x})$

(respectively $\left.T_{n}(x \alpha y)=\sum_{i=1}^{n} t_{i-1}(y) \alpha T_{i}(x)\right)$

\section{Example ( 2.2 )}

Let $\mathrm{T}=\left(\mathrm{T}_{\mathrm{i}}\right)_{\mathrm{i} \in \mathrm{N}}$ be a generalized higher reverse left (respectively right) centralizer of a ring $\mathrm{R}$, into itself associated with the higher reverse left (respectively right) centralizer $t=\left(t_{i}\right)_{i \in N}$ of $R$ such that, for all $x$, $\mathrm{y} \in \mathrm{R}$ and $\mathrm{n} \in \mathrm{N}:$

$T_{n}(x y)=\sum_{i=1}^{n} T_{i}(y) t_{i-1}(x)$

(respectively $\left.\mathbf{T}_{\mathrm{n}}(\mathrm{xy})=\sum_{\mathrm{i}=1}^{\mathrm{n}} \mathrm{t}_{\mathrm{i}-1}(\mathrm{y}) \mathrm{T}_{\mathrm{i}}(\mathrm{x})\right)$

Let $\mathrm{M}=\mathrm{M}_{1 \times 2}(\mathrm{R})$ and $\Gamma=\left\{\left(\begin{array}{l}\mathbf{n} \\ \mathbf{0}\end{array}\right), \mathbf{n} \in \mathbf{Z}\right\}$. Then $\mathrm{M}$ is a $\Gamma$-ring .

Let $\mathrm{F}=(\mathrm{Fi})_{\mathrm{i} \in \mathrm{N}}$ be a family of additive mappings from a $\Gamma$-ring $\mathrm{M}$ into itself, such that for all $(\mathrm{x} \quad \mathrm{y}) \in \mathrm{M}$ $\mathrm{F}_{\mathrm{n}}\left(\left(\begin{array}{ll}\mathrm{x} & \mathrm{y}\end{array}\right)\right)=\left(\begin{array}{cc}\mathrm{T}_{\mathrm{n}}(\mathrm{x}) & \mathrm{T}_{\mathrm{n}}(\mathrm{y})\end{array}\right)$.

Then, there exists a higher reverse left (respectively right) centralizer $f=\left(f_{i}\right)_{i \in N}$ of a $\Gamma$-ring $M$ into itself, such that for all $\left(\begin{array}{ll}x & y\end{array}\right) \in M$, the following equation holds :

$\mathrm{f}_{\mathrm{n}}\left(\left(\begin{array}{ll}\mathrm{x} & \mathrm{y}\end{array}\right)\right)=\left(\begin{array}{ll}\mathrm{t}_{\mathrm{n}}(\mathrm{x}) & \mathrm{t}_{\mathrm{n}}(\mathrm{y})\end{array}\right)$.

Therefore, $F_{n}$ is a generalized higher reverse left (respectively right) centralizer of $M$.

Definition ( 2.3 )

Let $\mathrm{T}=\left(\mathrm{T}_{\mathrm{i}}\right)_{\mathrm{i} \in \mathrm{N}}$ be a family of additive mappings of a $\Gamma$-ring $\mathrm{M}$ into itself .Then $\mathrm{T}$ is called a Jordan generalized higher reverse left (respectively right) centralizer of a $\Gamma$-ring $M$ associated with the Jordan higher 
reverse left(respectively right) centralizer $t=\left(t_{i}\right)_{i \in N}$ of the $\Gamma$-ring $M$ into itself, if for all $x \in M, \alpha \in \Gamma$ and $\mathrm{n} \in \mathrm{N}$, the following equation holds :

$\mathrm{T}(\mathrm{x} \alpha \mathrm{x})=\sum_{\mathrm{i}=1}^{\mathrm{n}} \mathrm{T}_{\mathrm{i}}(\mathrm{x}) \alpha \mathrm{t}_{\mathrm{i}-1}(\mathrm{x})$

(respectively $\left.T_{n}(x \alpha x)=\sum_{i=1}^{n} t_{i-1}(x) \alpha T_{i}(x)\right)$.

\section{Definition ( 2.4 )}

Let $\mathrm{T}=\left(\mathrm{T}_{\mathrm{i}}\right)_{\mathrm{i} \in \mathrm{N}}$ be a family of additive mappings of a $\Gamma$-ring $\mathrm{M}$ into itself .Then $\mathrm{T}$ is called a Jordan triple generalized higher reverse left (respectively right) centralizer of a $\Gamma$-ring M, associated with the Jordan triple higher reverse left (respectively right) centralizer $t=\left(t_{i}\right)_{i \in N}$ of the $\Gamma$-ring $M$ into itself if , for all $x, y \in$ $\mathrm{M}, \alpha, \beta \in \Gamma$ and $\mathrm{n} \in \mathrm{N}$, the following equation holds :

$\mathrm{T}_{\mathrm{n}}(\mathrm{x} \alpha \mathrm{y} \beta \mathrm{x})=\sum_{\mathrm{i}=1}^{\mathrm{n}} \mathrm{T}_{\mathrm{i}}(\mathrm{x}) \beta \mathrm{t}_{\mathrm{i}-1}(\mathrm{y}) \alpha \mathrm{t}_{\mathrm{i}-1}(\mathrm{x})$

(respectively resp. $\left.T_{n}(x \alpha y \beta x)=\sum_{i=1}^{n} t_{i-1}(x) \beta t_{i-1}(y) \alpha T_{i}(x)\right)$.

\section{Lemma ( 2.5$)$}

LetT $=\left(\mathrm{T}_{i}\right)_{\mathrm{i} \in \mathrm{N}}$ be a Jordan generalized higher reverse left (respectively right) centralizer of a $\Gamma$-ring $\mathrm{M}$ into itself. Then for all $\mathrm{x}, \mathrm{y}, \mathrm{z} \in \mathrm{M}, \alpha, \beta \in \Gamma$ and $\mathrm{n} \in \mathrm{N}$, the following equation holds :

(i) $\mathrm{T}_{\mathrm{n}}(\mathrm{x} \alpha \mathrm{y}+\mathrm{y} \alpha \mathrm{x})=\sum_{\mathrm{i}=1}^{\mathrm{n}} \mathrm{T}_{\mathrm{i}}(\mathrm{y}) \alpha \mathrm{t}_{\mathrm{i}-1}(\mathrm{x})+\sum_{\mathrm{i}=1}^{\mathrm{n}} \mathrm{T}_{\mathrm{i}}(\mathrm{x}) \alpha \mathrm{t}_{\mathrm{i}-1}(\mathrm{y})$

$\left(\right.$ respectively $\left.T_{n}(x \alpha y+y \alpha x)=\sum_{i=1}^{n} t_{i-1}(y) \alpha T_{i}(x)+\sum_{i=1}^{n} t_{i-1}(x) \alpha T_{i}(y)\right)$

(ii) $\mathrm{T}_{\mathrm{n}}(\mathrm{x} \alpha \mathrm{y} \beta \mathrm{x}+\mathrm{x} \beta \mathrm{y} \alpha \mathrm{x})=\sum_{\mathrm{i}=1}^{\mathrm{n}} \mathrm{T}_{\mathrm{i}}(\mathrm{x}) \beta \mathrm{t}_{\mathrm{i}-1}(\mathrm{y}) \alpha \mathrm{t}_{\mathrm{i}-1}(\mathrm{x})+\sum_{\mathrm{i}=1}^{\mathrm{n}} \mathrm{T}_{\mathrm{i}}(\mathrm{x}) \alpha \mathrm{t}_{\mathrm{i}-1}(\mathrm{y}) \beta \mathrm{t}_{\mathrm{i}-1}(\mathrm{x})$ $\left(\right.$ respectively $\left.\mathrm{T}_{\mathrm{n}}(\mathrm{x} \alpha \mathrm{y} \beta \mathrm{x}+\mathrm{x} \beta \mathrm{y} \alpha \mathrm{x})=\sum_{\mathrm{i}=1}^{\mathrm{n}} \mathrm{t}_{\mathrm{i}-1}(\mathrm{x}) \beta \mathrm{t}_{\mathrm{i}-1}(\mathrm{y}) \alpha \mathrm{T}_{\mathrm{i}}(\mathrm{x})+\sum_{\mathrm{i}=1}^{\mathrm{n}} \mathrm{t}_{\mathrm{i}-1}(\mathrm{x}) \alpha \mathrm{t}_{\mathrm{i}-1}(\mathrm{y}) \beta \mathrm{T}_{\mathrm{i}}(\mathrm{x})\right)$

(iii) $\mathrm{T}_{\mathrm{n}}(\mathrm{x} \alpha \mathrm{y} \beta \mathrm{z}+\mathrm{z} \alpha \mathrm{y} \beta \mathrm{x})=\sum_{\mathrm{i}=1}^{\mathrm{n}} \mathrm{T}_{\mathrm{i}}(\mathrm{z}) \boldsymbol{\beta} \mathrm{t}_{\mathrm{i}-1}(\mathrm{y}) \alpha \mathrm{t}_{\mathrm{i}-1}(\mathrm{x})+\sum_{\mathrm{i}=1}^{\mathrm{n}} \mathrm{T}_{\mathrm{i}}(\mathrm{x}) \beta \mathrm{t}_{\mathrm{i}-1}(\mathrm{y}) \alpha \mathrm{t}_{\mathrm{i}-1}(\mathrm{z})$ (respectively $\mathrm{T}_{\mathrm{n}}(\mathrm{x} \alpha \mathrm{y} \beta \mathrm{z}+\mathrm{z} \alpha \mathrm{y} \beta \mathrm{x})=\sum_{\mathrm{i}=1}^{\mathrm{n}} \mathrm{t}_{\mathrm{i}-1}(\mathrm{z}) \beta \mathrm{t}_{\mathrm{i}-1}(\mathrm{y}) \alpha \mathrm{T}_{\mathrm{i}}(\mathrm{x})+\sum_{\mathrm{i}=1}^{\mathrm{n}} \mathrm{t}_{\mathrm{i}-1}(\mathrm{x}) \beta \mathrm{t}_{\mathrm{i}-1}(\mathrm{y}) \alpha \mathrm{T}_{\mathrm{i}}(\mathrm{z})$ )

(iv) In particular, if $M$ is a 2-torsion free commutative $\Gamma$-ring, then

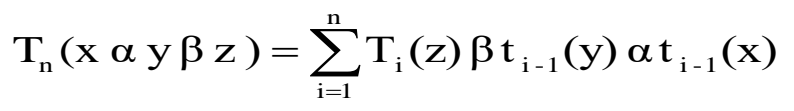

(respectively $\left.T_{n}(x \alpha y \beta z)=\sum_{i=1}^{n} t_{i-1}(z) \beta t_{i-1}(y) \alpha T_{i}(x)\right)$

$(\mathrm{v}) \mathrm{T}_{\mathrm{n}}(\mathrm{x} \alpha \mathrm{y} \alpha \mathrm{z}+\mathrm{z} \alpha \mathrm{y} \alpha \mathrm{x})=\sum_{\mathrm{i}=1}^{\mathrm{n}} \mathrm{T}_{\mathrm{i}}(\mathrm{z}) \alpha \mathrm{t}_{\mathrm{i}-1}(\mathrm{y}) \alpha \mathrm{t}_{\mathrm{i}-1}(\mathrm{x})+\sum_{\mathrm{i}=1}^{\mathrm{n}} \mathrm{T}_{\mathrm{i}}(\mathrm{x}) \alpha \mathrm{t}_{\mathrm{i}-1}(\mathrm{y}) \alpha \mathrm{t}_{\mathrm{i}-1}(\mathrm{z})$ $\left(\right.$ respectively $\left.\mathrm{T}_{\mathrm{n}}(\mathrm{x} \alpha \mathrm{y} \alpha \mathrm{z}+\mathrm{z} \alpha \mathrm{y} \alpha \mathrm{x})=\sum_{\mathrm{i}=1}^{\mathrm{n}} \mathrm{t}_{\mathrm{i}-1}(\mathrm{z}) \alpha \mathrm{t}_{\mathrm{i}-1}(\mathrm{y}) \alpha \mathrm{T}_{\mathrm{i}}(\mathrm{x})+\sum_{\mathrm{i}=1}^{\mathrm{n}} \mathrm{t}_{\mathrm{i}-1}(\mathrm{x}) \alpha \mathrm{t}_{\mathrm{i}-1}(\mathrm{y}) \alpha \mathrm{T}_{\mathrm{i}}(\mathrm{z})\right)$ 


\section{Proof:}

(i) Since $\mathrm{T}$ is Jordan generalized higher reverse left (respectively right) centralizer,

(ii) we have that

$$
\begin{aligned}
& \mathrm{T}_{\mathrm{n}}((\mathrm{x}+\mathrm{y}) \alpha(\mathrm{x}+\mathrm{y}))=\sum_{\mathrm{i}=1}^{\mathrm{n}} \mathrm{T}_{\mathrm{i}}(\mathrm{x}+\mathrm{y}) \alpha \mathrm{t}_{\mathrm{i}-1}(\mathrm{x}+\mathrm{y}) \\
& =\sum_{\mathrm{i}=1}^{\mathrm{n}} \mathrm{T}_{\mathrm{i}}(\mathrm{x}) \alpha \mathrm{t}_{\mathrm{i}-1}(\mathrm{x})+\sum_{\mathrm{i}=1}^{\mathrm{n}} \mathrm{T}_{\mathrm{i}}(\mathrm{x}) \alpha \mathrm{t}_{\mathrm{i}-1}(\mathrm{y})+\sum_{\mathrm{i}=1}^{\mathrm{n}} \mathrm{T}_{\mathrm{i}}(\mathrm{y}) \alpha \mathrm{t}_{\mathrm{i}-1}(\mathrm{x})+\sum_{\mathrm{i}=1}^{\mathrm{n}} \mathrm{T}_{\mathrm{i}}(\mathrm{y}) \alpha \mathrm{t}_{\mathrm{i}-1}(\mathrm{y})
\end{aligned}
$$

Meanwhile, we have that

$$
\begin{aligned}
& T_{n}((x+y) \alpha(x+y))=T_{n}(x \alpha x+x \alpha y+y \alpha x+y \alpha y) \\
& =T_{n}(x \alpha x)+T_{n}(y \alpha y)+T_{n}(x \alpha y+y \alpha x) \\
& =\sum_{i=1}^{n} T_{i}(x) \alpha t_{i-1}(x)+\sum_{i=1}^{n} T_{i}(y) \alpha t_{i-1}(y)+T_{n}(x \alpha y+y \alpha x)
\end{aligned}
$$

We obtain the following equation by Comparing equations ( 1 ) and (2)

$\mathrm{T}_{\mathrm{n}}(\mathrm{x} \alpha \mathrm{y}+\mathrm{y} \alpha \mathrm{x})=\sum_{\mathrm{i}=1}^{\mathrm{n}} \mathrm{T}_{\mathrm{i}}(\mathrm{y}) \alpha \mathrm{t}_{\mathrm{i}-1}(\mathrm{x})+\sum_{\mathrm{i}=1}^{\mathrm{n}} \mathrm{T}_{\mathrm{i}}(\mathrm{x}) \alpha \mathrm{t}_{\mathrm{i}-1}(\mathrm{y})$

(ii) By substituting that ), we have i (Lemma (2.5) in $b$ for ) $a \beta b+b \beta a$ (

$=\sum_{\mathrm{i}=1}^{\mathrm{n}} \mathrm{T}_{\mathrm{i}}(\mathrm{y}) \beta \mathrm{t}_{\mathrm{i}-1}(\mathrm{x}) \alpha \mathrm{t}_{\mathrm{i}-1}(\mathrm{x})+\mathrm{T}_{\mathrm{i}}(\mathrm{x}) \beta \mathrm{t}_{\mathrm{i}-1}(\mathrm{y}) \alpha \mathrm{t}_{\mathrm{i}-1}(\mathrm{x})+$

$T_{i}(x) \alpha t_{i-1}(y) \beta t_{i-1}(x)+T_{i}(x) \alpha t_{i-1}(x) \beta t_{i-1}(y) \ldots(1)$

In addition, we obtain that

$\mathrm{T}_{\mathrm{n}}(\mathrm{x} \alpha(\mathrm{x} \beta \mathrm{y}+\mathrm{y} \beta \mathrm{x})+(\mathrm{x} \beta \mathrm{y}+\mathrm{y} \beta \mathrm{x}) \alpha \mathrm{x})$

$=\mathrm{T}_{\mathrm{n}}(\mathrm{x} \alpha \mathrm{x} \beta \mathrm{y}+\mathrm{x} \alpha \mathrm{y} \beta \mathrm{x}+\mathrm{x} \beta \mathrm{y} \alpha \mathrm{x}+\mathrm{y} \beta \mathrm{x} \alpha \mathrm{x})$

$=T_{n}(y \beta \alpha x)+T_{n}(x \alpha \beta y)+T_{n}(x \alpha y \beta x+x \beta y \alpha x)$

$=\sum_{i=1}^{n} T_{i}(y) \beta t_{i-1}(x) \alpha t_{i-1}(x)+T_{i}(x) \alpha t_{i-1}(x) \beta t_{i-1}(y)+T_{n}(x \alpha y \beta x+x \beta y \alpha x)$

We get the following equation by Comparing equations ( 1 ) and ( 2 )

$T_{n}(x \alpha y \beta x+x \beta y \alpha x)=\sum_{i=1}^{n} T_{i}(x) \beta t_{i-1}(y) \alpha t_{i-1}(x)+\sum_{i=1}^{n} T_{i}(x) \alpha t_{i-1}(y) \beta t_{i-1}(x)$

(iii) By substituting $(a+c)$ for $a$ in Definition (2.4), we have

$$
\begin{aligned}
& =\sum_{\mathrm{i}=1}^{\mathrm{n}} \mathrm{T}_{\mathrm{i}}(\mathrm{x}) \beta \mathrm{t}_{\mathrm{i}-1}(\mathrm{y}) \alpha \mathrm{t}_{\mathrm{i}-1}(\mathrm{x})+\mathrm{T}_{\mathrm{i}}(\mathrm{x}) \beta \mathrm{t}_{\mathrm{i}-1}(\mathrm{y}) \alpha \mathrm{t}_{\mathrm{i}-1}(\mathrm{z})+ \\
& \mathrm{T}_{\mathrm{i}}(\mathrm{z}) \beta \mathrm{t}_{\mathrm{i}-1}(\mathrm{y}) \alpha \mathrm{t}_{\mathrm{i}-1}(\mathrm{x})+\mathrm{T}_{\mathrm{i}}(\mathrm{z}) \beta \mathrm{t}_{\mathrm{i}-1}(\mathrm{y}) \alpha \mathrm{t}_{\mathrm{i}-1}(\mathrm{z}) \ldots(1)
\end{aligned}
$$

\section{Moreover,}

$\mathrm{T}_{\mathrm{n}}((\mathrm{x}+\mathrm{z}) \alpha \mathrm{y} \beta(\mathrm{x}+\mathrm{z}))=\mathrm{T}_{\mathrm{n}}(\mathrm{x} \alpha \mathrm{y} \beta \mathrm{x}+\mathrm{x} \alpha \mathrm{y} \beta \mathrm{z}+\mathrm{z} \alpha \mathrm{y} \beta \mathrm{x}+\mathrm{z} \alpha \mathrm{y} \beta \mathrm{z})$

$T_{n}(x \alpha y \beta x)+T_{n}(z \alpha y \beta z)+T_{n}(x \alpha y \beta z+z \alpha y \beta x)$ 
$=\sum_{\mathrm{i}=1}^{\mathrm{n}} \mathrm{T}_{\mathrm{i}}(\mathrm{x}) \boldsymbol{\beta} \mathrm{t}_{\mathrm{i}-1}(\mathrm{y}) \alpha \mathrm{t}_{\mathrm{i}-1}(\mathrm{x})+\mathrm{T}_{\mathrm{i}}(\mathrm{z}) \beta \mathrm{t}_{\mathrm{i}-1}(\mathrm{y}) \alpha \mathrm{t}_{\mathrm{i}-1}(\mathrm{z}) \mathrm{T}_{\mathrm{n}}(\mathrm{x} \alpha \mathrm{y} \beta \mathrm{z}+\mathrm{z} \alpha \mathrm{y} \boldsymbol{\mathrm { x }})$

The following equation is obtained by comparing equations ( 1 ) and ( 2 )

$T_{n}(x \alpha y \beta z+z \alpha y \beta x)=\sum_{i=1}^{n} T_{i}(z) \beta t_{i-1}(y) \alpha t_{i-1}(x)+\sum_{i=1}^{n} T_{i}(x) \beta t_{i-1}(y) \alpha t_{i-1}(z)$

(iv) Using Lemma ( 2.5 ) ( iii ) and the fact that $\mathrm{M}$ is a commutative $\Gamma$-ring, we have that

$$
\begin{aligned}
& \mathrm{T}_{\mathrm{n}}(\mathrm{x} \alpha \mathrm{y} \beta \mathrm{z}+\mathrm{x} \alpha \mathrm{y} \beta \mathrm{z})=2 \mathrm{~T}_{\mathrm{n}}(\mathrm{x} \alpha \mathrm{y} \beta \mathrm{z}) \\
& =2 \sum_{\mathrm{i}=1}^{\mathrm{n}} \mathrm{T}_{\mathrm{i}}(\mathrm{z}) \beta \mathrm{t}_{\mathrm{i}-1}(\mathrm{y}) \alpha \mathrm{t}_{\mathrm{i}-1}(\mathrm{x})
\end{aligned}
$$

We obtain the required result, by utilizing the fact that $\mathrm{M}$ is a 2-torsion free .

(v) The substitution $\beta$ for $\alpha$ in Lemma (2.5) (iii ), gives that

$$
\mathrm{T}_{\mathrm{n}}(\mathrm{x} \alpha \mathrm{y} \alpha \mathrm{z}+\mathrm{z} \alpha \mathrm{y} \alpha \mathrm{x})=\sum_{\mathrm{i}=1}^{\mathrm{n}} \mathrm{T}_{\mathrm{i}}(\mathrm{z}) \alpha \mathrm{t}_{\mathrm{i}-1}(\mathrm{y}) \alpha \mathrm{t}_{\mathrm{i}-1}(\mathrm{x})+\sum_{\mathrm{i}=1}^{\mathrm{n}} \mathrm{T}_{\mathrm{i}}(\mathrm{x}) \alpha \mathrm{t}_{\mathrm{i}-1}(\mathrm{y}) \alpha \mathrm{t}_{\mathrm{i}-1}(\mathrm{z})
$$

\section{Definition ( 2.6 )}

Let $\mathrm{T}=\left(\mathrm{T}_{\mathrm{i}}\right)_{\mathrm{i} \in \mathrm{N}}$ be a Jordan generalized higher reverse left (respectively right) centralizer of a $\Gamma$-ring $\mathrm{M}$ ring into itself . Then for all $\mathrm{x}, \mathrm{y} \in \mathrm{M}, \alpha \in \Gamma$ and $\mathrm{n} \in \mathrm{N}$, we define

$\delta_{\mathrm{n}}(\mathrm{x}, \mathrm{y})_{\alpha}=\mathrm{T}_{\mathrm{n}}(\mathrm{x} \alpha \mathrm{y})-\sum_{\mathrm{i}=1}^{\mathrm{n}} \mathrm{T}_{\mathrm{i}}(\mathrm{y}) \alpha \mathrm{t}_{\mathrm{i}-1}(\mathrm{x})$

$\left(\right.$ respectively $\left.\delta_{\mathrm{n}}(\mathrm{x}, \mathrm{y})_{\alpha}=\mathrm{T}_{\mathrm{n}}(\mathrm{x} \alpha \mathrm{y})-\sum_{\mathrm{i}=1}^{\mathrm{n}} \mathrm{t}_{\mathrm{i}-1}(\mathrm{y}) \alpha \mathrm{T}_{\mathrm{i}}(\mathrm{x})\right)$

\section{Lemma ( 2.7 )}

Let $\mathrm{T}=\left(\mathrm{T}_{\mathrm{i}}\right)_{\mathrm{i} \in \mathrm{N}}$ be a Jordan generalized higher reverse left (respectively right) centralizer of a $\Gamma$-ring $\mathrm{M}$ ring into itself .Then for all $\mathrm{x}, \mathrm{y}, \mathrm{z} \in \mathrm{M}, \alpha, \beta \in \Gamma$ and $\mathrm{n} \in \mathrm{N}$, we have that the following equations hold :

(i) $\delta_{\mathrm{n}}(\mathrm{x}, \mathrm{y})_{\alpha}=-\delta_{\mathrm{n}}(\mathrm{y}, \mathrm{x})_{\alpha}$

(ii) $\delta_{\mathrm{n}}(\mathrm{x}+\mathrm{y}, \mathrm{z})_{\alpha}=\delta_{\mathrm{n}}(\mathrm{x}, \mathrm{z})_{\alpha}+\delta_{\mathrm{n}}(\mathrm{y}, \mathrm{z})_{\alpha}$

(iii) $\delta_{\mathrm{n}}(\mathrm{x}, \mathrm{y}+\mathrm{z})_{\alpha}=\delta_{\mathrm{n}}(\mathrm{x}, \mathrm{y})_{\alpha}+\delta_{\mathrm{n}}(\mathrm{x}, \mathrm{z})_{\alpha}$

(iv) $\delta_{\mathrm{n}}(\mathrm{x}, \mathrm{y})_{\alpha+\beta}=\delta_{\mathrm{n}}(\mathrm{x}, \mathrm{y})_{\alpha}+\delta_{\mathrm{n}}(\mathrm{x}, \mathrm{y})_{\beta}$

\section{Proof:}

(i) By applying Lemma ( 2.5 ) ( i ), we have that

$$
\begin{aligned}
& \mathrm{T}_{\mathrm{n}}(\mathrm{x} \alpha \mathrm{y}+\mathrm{y} \alpha \mathrm{x})=\sum_{\mathrm{i}=1}^{\mathrm{n}} \mathrm{T}_{\mathrm{i}}(\mathrm{y}) \alpha \mathrm{t}_{\mathrm{i}-1}(\mathrm{x})+\sum_{\mathrm{i}=1}^{\mathrm{n}} \mathrm{T}_{\mathrm{i}}(\mathrm{x}) \alpha \mathrm{t}_{\mathrm{i}-1}(\mathrm{y}) \\
& \mathrm{T}_{\mathrm{n}}(\mathrm{x} \alpha \mathrm{y})-\sum_{\mathrm{i}=1}^{\mathrm{n}} \mathrm{T}_{\mathrm{i}}(\mathrm{y}) \alpha \mathrm{t}_{\mathrm{i}-1}(\mathrm{x})=-\left(\mathrm{T}_{\mathrm{n}}(\mathrm{y} \alpha \mathrm{x})-\sum_{\mathrm{i}=1}^{\mathrm{n}} \mathrm{T}_{\mathrm{i}}(\mathrm{x}) \alpha \mathrm{t}_{\mathrm{i}-1}(\mathrm{y})\right)
\end{aligned}
$$

Thus, $\delta_{\mathrm{n}}(\mathrm{x}, \mathrm{y})_{\alpha}=-\delta_{\mathrm{n}}(\mathrm{y}, \mathrm{x})_{\alpha}$ 
(ii) $\delta_{\mathrm{n}}(\mathrm{x}+\mathrm{y}, \mathrm{z})_{\alpha}=\mathrm{T}_{\mathrm{n}}((\mathrm{x}+\mathrm{y}) \alpha \mathrm{z})=\sum_{\mathrm{i}=1}^{\mathrm{n}} \mathrm{T}_{\mathrm{i}}(\mathrm{z}) \alpha \mathrm{t}_{\mathrm{i}-1}(\mathrm{x}+\mathrm{y})$

$=T_{n}(x \alpha z+y \alpha z)-\sum_{i=1}^{n} T_{i}(z) \alpha t_{i-1}(x)-\sum_{i=1}^{n} T_{i}(z) \alpha t_{i-1}(y)$

$=T_{n}(x \alpha z)-\sum_{i=1}^{n} T_{i}(z) \alpha t_{i-1}(x)+T_{n}(y \alpha z)-\sum_{i=1}^{n} T_{i}(z) \alpha t_{i-1}(y)$

$=\delta_{\mathrm{n}}(\mathrm{x}, \mathrm{z})_{\alpha}+\delta_{\mathrm{n}}(\mathrm{y}, \mathrm{z})_{\alpha}$

(iii) $\delta_{\mathrm{n}}(\mathrm{x}, \mathrm{y}+\mathrm{z})_{\alpha}=\mathrm{T}_{\mathrm{n}}(\mathrm{x} \alpha(\mathrm{y}+\mathrm{z}))=\sum_{\mathrm{i}=1}^{\mathrm{n}} \mathrm{T}_{\mathrm{i}}(\mathrm{y}+\mathrm{z}) \alpha \mathrm{t}_{\mathrm{i}-1}(\mathrm{x})$

$=T_{n}(x \alpha y+x \alpha z)-\sum_{i=1}^{n} T_{i}(y) \alpha t_{i-1}(x)-\sum_{i=1}^{n} T_{i}(z) \alpha t_{i-1}(x)$

$=T_{n}(x \alpha y)-\sum_{i=1}^{n} T_{i}(y) \alpha t_{i-1}(x)+T_{n}(x \alpha z)-\sum_{i=1}^{n} T_{i}(z) \alpha t_{i-1}(x)$

$=\delta_{\mathrm{n}}(\mathrm{x}, \mathrm{y})_{\alpha}+\delta_{\mathrm{n}}(\mathrm{x}, \mathrm{z})_{\alpha}$

(iv) $\delta_{\mathrm{n}}(\mathrm{x}, \mathrm{y})_{\alpha+\beta}=\mathrm{T}_{\mathrm{n}}(\mathrm{x}(\alpha+\beta) \mathrm{y})-\sum_{\mathrm{i}=1}^{\mathrm{n}} \mathrm{T}_{\mathrm{i}}(\mathrm{y})(\alpha+\beta) \mathrm{t}_{\mathrm{i}-1}(\mathrm{x})$

$=T_{n}(x \alpha y)-\sum_{i=1}^{n} T_{i}(y) \alpha t_{i-1}(x)+T_{n}(x \beta y)-\sum_{i=1}^{n} T_{i}(y) \beta t_{i-1}(x)$

$=\delta_{\mathrm{n}}(\mathrm{x}, \mathrm{y})_{\alpha}+\delta_{\mathrm{n}}(\mathrm{x}, \mathrm{y}) \beta$

Remark ( 2.8 )

It is noteworthy that $T=\left(T_{i}\right)_{i \in N}$ is a generalized higher reverse left (respectively right) centralizer of a $\Gamma$-ring $\mathrm{M}$, into itself if and only if $\delta_{\mathrm{n}}(\mathrm{x}, \mathrm{y})_{\alpha}=0$, for all $\mathrm{x}, \mathrm{y} \in \mathrm{M}, \alpha \in \Gamma$ and $\mathrm{n} \in \mathrm{N}$.

\section{Lemma ( 2.9)}

Let $T=\left(T_{i}\right)_{i \in N}$ be a Jordan generalized higher reverse left (respectively right) centralizer of a prime $\Gamma$-ring

$\mathrm{M}$, into itself. Then for all $\mathrm{x}, \mathrm{y}, \mathrm{z} \in \mathrm{M}, \alpha, \beta \in \Gamma$ and $\mathrm{n} \in \mathrm{N}$, the following equations hold :

(i) $\delta_{\mathrm{n}}(\mathrm{x}, \mathrm{y})_{\alpha} \beta \mathrm{t}_{\mathrm{n}-1}(\mathrm{z}) \beta\left[\mathrm{t}_{\mathrm{n}-1}(\mathrm{x}), \mathrm{t}_{\mathrm{n}-1}(\mathrm{y})\right]_{\alpha}=0$

(ii) $\delta_{\mathrm{n}}(\mathrm{x}, \mathrm{y})_{\alpha} \propto \mathrm{t}_{\mathrm{n}-1}(\mathrm{z}) \alpha\left[\mathrm{t}_{\mathrm{n}-1}(\mathrm{x}), \mathrm{t}_{\mathrm{n}-1}(\mathrm{y})\right]_{\alpha}=0$

(iii) $\delta_{\mathrm{n}}(\mathrm{x}, \mathrm{y})_{\beta} \alpha \mathrm{t}_{\mathrm{n}-1}(\mathrm{z}) \alpha\left[\mathrm{t}_{\mathrm{n}-1}(\mathrm{x}), \mathrm{t}_{\mathrm{n}-1}(\mathrm{y})\right]_{\beta}=0$

\section{Proof:}

(i) The Proof is utilizing induction on $\mathrm{n} \in \mathrm{N}$

If $\mathrm{n}=1$,

let let $\mathrm{w}=\mathrm{x} \alpha \mathrm{y} \beta \mathrm{z} \beta \mathrm{y} \alpha \mathrm{x}+\mathrm{y} \alpha \mathrm{x} \beta \mathrm{z} \beta \mathrm{x} \alpha \mathrm{y}$

Then, we obtain that

$$
\begin{aligned}
\mathrm{T}(\mathrm{w}) & =\mathrm{T}(\mathrm{x} \alpha(\mathrm{y} \beta \mathrm{z} \beta \mathrm{y}) \alpha \mathrm{x}+\mathrm{y} \alpha(\mathrm{x} \beta \mathrm{z} \beta \mathrm{x}) \alpha \mathrm{y}) \\
& =\mathrm{T}(\mathrm{x}) \alpha \mathrm{y} \beta \mathrm{z} \beta \mathrm{y} \alpha \mathrm{x}+\mathrm{t}(\mathrm{y}) \alpha \mathrm{x} \beta \mathrm{z} \beta \mathrm{x} \alpha \mathrm{y}
\end{aligned}
$$

Moreover, we have that

$$
\begin{aligned}
\mathrm{T}(\mathrm{w}) & =\mathrm{T}((\mathrm{x} \alpha \mathrm{y}) \beta \mathrm{z} \beta(\mathrm{y} \alpha \mathrm{x})+(\mathrm{y} \alpha \mathrm{x}) \beta \mathrm{z} \beta(\mathrm{x} \alpha \mathrm{y})) \\
& =\mathrm{T}(\mathrm{y} \alpha \mathrm{x}) \beta \mathrm{z} \beta \mathrm{y} \alpha \mathrm{x}+\mathrm{t}(\mathrm{x} \alpha \mathrm{y}) \beta \mathrm{z} \beta \mathrm{x} \alpha \mathrm{y}
\end{aligned}
$$


The Comparison of equations ( 1 ) and ( 2 ) yields that

$0=(\mathrm{T}(\mathrm{y} \alpha \mathrm{x})-\mathrm{t}(\mathrm{x}) \alpha \mathrm{y}) \beta \mathrm{z} \beta \mathrm{y} \alpha \mathrm{x}+(\mathrm{T}(\mathrm{x} \alpha \mathrm{y})-\mathrm{t}(\mathrm{y}) \alpha \mathrm{x}) \beta \mathrm{z} \beta \mathrm{x} \alpha \mathrm{y}$

$0=\delta(\mathrm{y}, \mathrm{x})_{\alpha} \beta \mathrm{z} \beta \mathrm{y} \alpha \mathrm{x}+\delta(\mathrm{x}, \mathrm{y})_{\alpha} \beta \mathrm{z} \beta \mathrm{x} \alpha \mathrm{y}$

$0=-\delta(\mathrm{x}, \mathrm{y})_{\alpha} \beta \mathrm{z} \beta \mathrm{y} \alpha \mathrm{x}+\delta(\mathrm{x}, \mathrm{y})_{\alpha} \beta \mathrm{z} \beta \mathrm{x} \alpha \mathrm{y}$

$0=\delta(\mathrm{x}, \mathrm{y})_{\alpha} \beta \mathrm{z} \beta(\mathrm{x} \alpha \mathrm{y}-\mathrm{y} \alpha \mathrm{x})$

Thus, $\delta(\mathrm{x}, \mathrm{y})_{\alpha} \beta \mathrm{z} \beta[\mathrm{x}, \mathrm{y}]_{\alpha}=0$, for all $\mathrm{x}, \mathrm{y}, \mathrm{z} \in \mathrm{M}$ and $\alpha, \beta \in \Gamma$.

Now, we can ssume the following :

$\delta_{\mathrm{s}}(\mathrm{x}, \mathrm{y})_{\alpha} \beta \mathrm{t}_{\mathrm{s}-1}(\mathrm{z}) \beta\left[\mathrm{t}_{\mathrm{s}-1}(\mathrm{x}), \mathrm{t}_{\mathrm{s}-1}(\mathrm{y})\right]_{\alpha}=0$, for all $\mathrm{x}, \mathrm{y}, \mathrm{z} \in \mathrm{M}$, and

$, \mathrm{n} \in \mathrm{N}, \mathrm{s}<\mathrm{n}$.

$\mathrm{T}_{\mathrm{n}}(\mathrm{w})=\mathrm{T}_{\mathrm{n}}(\mathrm{x} \alpha(\mathrm{y} \beta \mathrm{z} \beta \mathrm{y}) \alpha \mathrm{x}+\mathrm{y} \alpha(\mathrm{x} \beta \mathrm{z} \beta \mathrm{x}) \alpha \mathrm{y})$

$=\sum_{i=1}^{n} T_{i}(x) \alpha t_{i-1}(y \beta z y) \alpha t_{i-1}(x)+\sum_{i=1}^{n} T_{i}(y) \alpha t_{i-1}(x \beta z \beta x) \alpha t_{i-1}(y)$

$=\sum_{i=1}^{n} T_{i}(x) \alpha t_{i-1}(y) \beta t_{i-1}(z) \beta t_{i-1}(y) \alpha t_{i-1}(x)+$

${ }_{\mathrm{i}} \mathrm{t} \alpha(\mathrm{y}) \mathrm{iT} \sum_{\mathrm{i}=1}^{\mathrm{n}}$

${ }_{-1}(\mathrm{x}) \beta \mathrm{t}_{\mathrm{i}-1}(\mathrm{z}) \beta \mathrm{t}_{\mathrm{i}-1}(\mathrm{x}) \alpha \mathrm{t}_{\mathrm{i}-1}(\mathrm{y})$

$=\left(\sum_{\mathrm{i}=1}^{\mathrm{n}} \mathrm{T}_{\mathrm{i}}(\mathrm{x}) \alpha \mathrm{t}_{\mathrm{i}-1}(\mathrm{y})\right) \beta \mathrm{t}_{\mathrm{n}-1}(\mathrm{z}) \beta \mathrm{t}_{\mathrm{n}-1}(\mathrm{y}) \alpha \mathrm{t}_{\mathrm{n}-1}(\mathrm{x})+_{1-\mathrm{i}} \mathrm{t} \alpha(\mathrm{x})_{\mathrm{i}} \mathrm{T} \sum_{\mathrm{i}=1}^{\mathrm{n}-1}$

(y) $\beta \mathrm{t}_{\mathrm{i-1}}(\mathrm{z}) \beta \mathrm{t}_{\mathrm{i}-1}(\mathrm{y}) \alpha \mathrm{t}_{\mathrm{i}-1}(\mathrm{x})+$

$\left(\sum_{i=1}^{n} \quad T_{i}(y) \alpha t_{i-1}(x)\right) \beta t_{n-1}(z) \beta t_{n-1}(x) \alpha t_{n-1}(y)+\alpha(y)_{i} T \sum_{i=1}^{n-1}$

$t_{i-1}(x) \beta t_{i-1}(z) \beta t_{i-1}(x) \alpha t_{i-1}(y)$

Thus,

$\mathrm{T}_{\mathrm{n}}(\mathrm{w})=\mathrm{T}_{\mathrm{n}}((\mathrm{x} \alpha \mathrm{y}) \beta \mathrm{z} \beta(\mathrm{y} \alpha \mathrm{x})+(\mathrm{y} \alpha \mathrm{x}) \beta \mathrm{z} \beta(\mathrm{x} \alpha \mathrm{y}))$

$=\sum_{i=1}^{n} T_{i}(y \alpha x) \beta t_{i-1}(z) \beta t_{i-1}(x \alpha y)+\sum_{i=1}^{n} T_{i}(x \alpha y) \beta t_{i-1}(z) \beta t_{i-1}(y \alpha x)$

$=\mathrm{T}_{\mathrm{n}}(\mathrm{y} \alpha \mathrm{x}) \beta \mathrm{t}_{\mathrm{n}-1}(\mathrm{z}) \beta \mathrm{t}_{\mathrm{n}-1}(\mathrm{y}) \alpha \mathrm{t}_{\mathrm{n}-1}(\mathrm{x})+\sum_{\mathrm{i}=1}^{\mathrm{n}-1} \mathrm{~T}_{\mathrm{i}}(\mathrm{y} \alpha \mathrm{x}) \beta \mathrm{t}_{\mathrm{i}-1}(\mathrm{z}) \beta \mathrm{t}_{\mathrm{i}-1}(\mathrm{y})$

$\alpha \mathrm{t}_{\mathrm{i}-1}(\mathrm{x})+\mathrm{T}_{\mathrm{n}}(\mathrm{x} \alpha \mathrm{y}) \beta \mathrm{t}_{\mathrm{n}-1}(\mathrm{z}) \beta \mathrm{t}_{\mathrm{n}-1}(\mathrm{x}) \alpha \mathrm{t}_{\mathrm{n}-1}(\mathrm{y})$

$\sum_{i=1}^{n-1} T_{i}(x \alpha y) \beta t_{i-1}(z) \beta t_{i-1}(x) \alpha t_{i-1}(y)$

By comparing equations ( 3 ) and ( 4 ), we have that $0=\left(\mathrm{T}_{\mathrm{n}}(\mathrm{y} \alpha \mathrm{x})-\sum_{\mathrm{i}=1}^{\mathrm{n}} \mathrm{T}_{\mathrm{i}}(\mathrm{x}) \alpha \mathrm{t}_{\mathrm{i}-1}(\mathrm{y})\right) \beta \mathrm{t}_{\mathrm{n}-1}(\mathrm{z}) \beta \mathrm{t}_{\mathrm{n}-1}(\mathrm{y}) \alpha \mathrm{t}_{\mathrm{n}-1}(\mathrm{x})+$ 


$$
\begin{aligned}
& \left(\mathrm{T}_{\mathrm{n}}(\mathrm{x} \alpha \mathrm{y})-\sum_{\mathrm{i}=1}^{\mathrm{n}} \mathrm{T}_{\mathrm{i}}(\mathrm{y}) \alpha \mathrm{t}_{\mathrm{i}-1}(\mathrm{x})\right) \beta \mathrm{t}_{\mathrm{n}-1}(\mathrm{z}) \beta \mathrm{t}_{\mathrm{n}-1}(\mathrm{x}) \alpha \mathrm{t}_{\mathrm{n}-1}(\mathrm{y})+ \\
& \left.+)\left(\mathrm{x}_{1-\mathrm{i}} \mathrm{t} \alpha(\mathrm{y})_{1-\mathrm{i}} \mathrm{t} \beta(\mathrm{z})_{1-\mathrm{i}} \mathrm{t} \beta\right) \mathrm{y}\right)\left({ }_{1-\mathrm{i}} \mathrm{t} \alpha(\mathrm{x})_{\mathrm{i}} \mathrm{T}-\right) \mathrm{x} \alpha \mathrm{y}\left({ } _ { \mathrm { i } } \mathrm { T } \left(\sum_{\mathrm{i}=1}^{\mathrm{n}-1}\right.\right. \\
& \sum_{\mathrm{i}=1}^{\mathrm{n}-1}\left(\mathrm{~T}_{\mathrm{i}}(\mathrm{x} \alpha \mathrm{y})-\mathrm{T}_{\mathrm{i}}(\mathrm{y}) \alpha \mathrm{t}_{\mathrm{i}-1}(\mathrm{x})\right) \beta \mathrm{t}_{\mathrm{i}-1}(\mathrm{z}) \beta \mathrm{t}_{\mathrm{i}-1}(\mathrm{x}) \alpha \mathrm{t}_{\mathrm{i}-1}(\mathrm{y}) .
\end{aligned}
$$

It follows that

$$
\begin{aligned}
0= & \delta_{\mathrm{n}}(\mathrm{y}, \mathrm{x})_{\alpha} \beta \mathrm{t}_{\mathrm{n}-1}(\mathrm{z}) \beta \mathrm{t}_{\mathrm{n}-1}(\mathrm{y}) \alpha \mathrm{t}_{\mathrm{n}-1}(\mathrm{x})+ \\
& \delta_{\mathrm{n}}(\mathrm{x}, \mathrm{y})_{\alpha} \beta \mathrm{t}_{\mathrm{n}-1}(\mathrm{z}) \beta \mathrm{t}_{\mathrm{n}-1}(\mathrm{x}) \alpha \mathrm{t}_{\mathrm{n}-1}(\mathrm{y})
\end{aligned}
$$

$0=+$

$$
\mathrm{x})\left(\text { 1-n }^{\mathrm{t}} \alpha(\mathrm{y})_{1-\mathrm{n}} \mathrm{t} \beta(\mathrm{z})_{1-\mathrm{n}} \mathrm{t} \beta_{\alpha}(\mathrm{x}, \mathrm{y})_{\mathrm{n}} \delta-\right.
$$

$$
\delta_{\mathrm{n}}(\mathrm{x}, \mathrm{y})_{\alpha} \beta \mathrm{t}_{\mathrm{n}-1}(\mathrm{z}) \beta \mathrm{t}_{\mathrm{n}-1}(\mathrm{x}) \alpha \mathrm{t}_{\mathrm{n}-1}(\mathrm{y})
$$

$0=\delta_{\mathrm{n}}(\mathrm{x}, \mathrm{y})_{\alpha} \beta \mathrm{t}_{\mathrm{n}-1}(\mathrm{z}) \beta\left(\mathrm{t}_{\mathrm{n}-1}(\mathrm{x}) \alpha \mathrm{t}_{\mathrm{n}-1}(\mathrm{y})-\mathrm{t}_{\mathrm{n}-1}(\mathrm{y}) \alpha \mathrm{t}_{\mathrm{n}-1}(\mathrm{x})\right)$

Thus, $\delta_{\mathrm{n}}(\mathrm{x}, \mathrm{y})_{\alpha} \beta \mathrm{t}_{\mathrm{n}-1}(\mathrm{z}) \beta\left[\mathrm{t}_{\mathrm{n}-1}(\mathrm{x}), \mathrm{t}_{\mathrm{n}-1}(\mathrm{y})\right]_{\alpha}=0$, for all $\mathrm{x}, \mathrm{y}, \mathrm{z} \in \mathrm{M}$,

$\alpha, \beta \in \Gamma$ and $n \in N$

(ii) By substituting $\beta$ for $\alpha$ in Lemma ( 2.9 ) ( i ) and applying similar arguments as in the proof of Lemma ( 2.9 ) ( i ), we obtain Lemma ( 2.9 ) ( ii ) .

(iii) We get Lemma ( 2.9 ) ( iii ), by Interchanging $\alpha$ and $\beta$ in Lemma ( 2.9 ) ( i ) .

\section{Lemma ( 2.10 )}

Let $\mathrm{T}=\left(\mathrm{T}_{\mathrm{i}}\right)_{\mathrm{i} \in \mathrm{N}}$ be a Jordan generalized higher reverse left (respectively right) centralizer of a prime $\Gamma$-ring

$\mathrm{M}$ into itself .Then for all $\mathrm{x}, \mathrm{y}, \mathrm{z}, \mathrm{u}, \mathrm{v} \in \mathrm{M}$, $\alpha, \beta \in \Gamma$ and $\mathrm{n} \in \mathrm{N}$

(i) $\delta_{\mathrm{n}}(\mathrm{x}, \mathrm{y})_{\alpha} \beta \mathrm{t}_{\mathrm{n}-1}(\mathrm{z}) \beta\left[\mathrm{t}_{\mathrm{n}-1}(\mathrm{u}), \mathrm{t}_{\mathrm{n}-1}(\mathrm{v})\right]_{\alpha}=0$

(ii) $\delta_{\mathrm{n}}(\mathrm{x}, \mathrm{y})_{\alpha} \alpha \mathrm{t}_{\mathrm{n}-1}(\mathrm{z}) \alpha\left[\mathrm{t}_{\mathrm{n}-1}(\mathrm{u}), \mathrm{t}_{\mathrm{n}-1}(\mathrm{v})\right]_{\alpha}=0$

(iii) $\delta_{\mathrm{n}}(\mathrm{x}, \mathrm{y})_{\alpha} \alpha \mathrm{t}_{\mathrm{n}-1}(\mathrm{z}) \alpha\left[\mathrm{t}_{\mathrm{n}-1}(\mathrm{u}), \mathrm{t}_{\mathrm{n}-1}(\mathrm{v})\right]_{\beta}=0$

\section{Proof:}

(i) By substituting $(a+c)$ for $a$ in Lemma (2.9) (i ), we have that $\delta_{\mathrm{n}}(\mathrm{x}+\mathrm{u}, \mathrm{y})_{\alpha} \beta \mathrm{t}_{\mathrm{n}-1}(\mathrm{z}) \beta\left[\mathrm{t}_{\mathrm{n}-1}(\mathrm{x}+\mathrm{u}), \mathrm{t}_{\mathrm{n}-1}(\mathrm{y})\right]_{\alpha}=0$. Thus

$\delta_{\mathrm{n}}(\mathrm{x}, \mathrm{y})_{\alpha} \beta \mathrm{t}_{\mathrm{n}-1}(\mathrm{z}) \beta\left[\mathrm{t}_{\mathrm{n}-1}(\mathrm{x}), \mathrm{t}_{\mathrm{n}-1}(\mathrm{y})\right]_{\alpha}+$

$\delta_{\mathrm{n}}(\mathrm{x}, \mathrm{y})_{\alpha} \beta \mathrm{t}_{\mathrm{n}-1}(\mathrm{z}) \beta\left[\mathrm{t}_{\mathrm{n}-1}(\mathrm{u}), \mathrm{t}_{\mathrm{n}-1}(\mathrm{y})\right]_{\alpha}+$

$\delta_{\mathrm{n}}(\mathrm{u}, \mathrm{y})_{\alpha} \beta \mathrm{t}_{\mathrm{n}-1}(\mathrm{z}) \beta\left[\mathrm{t}_{\mathrm{n}-1}(\mathrm{x}), \mathrm{t}_{\mathrm{n}-1}(\mathrm{y})\right]_{\alpha}+$

$\delta_{\mathrm{n}}(\mathrm{u}, \mathrm{y})_{\alpha} \beta \mathrm{t}_{\mathrm{n}-1}(\mathrm{z}) \beta\left[\mathrm{t}_{\mathrm{n}-1}(\mathrm{u}), \mathrm{t}_{\mathrm{n}-1}(\mathrm{y})\right]_{\alpha}=0$

By applying Lemma ( 2.9 ) ( i ), we obtain that

$$
\begin{aligned}
& \delta_{\mathrm{n}}(\mathrm{x}, \mathrm{y})_{\alpha} \beta \mathrm{t}_{\mathrm{n}-1}(\mathrm{z}) \beta\left[\mathrm{t}_{\mathrm{n}-1}(\mathrm{u}), \mathrm{t}_{\mathrm{n}-1}(\mathrm{y})\right]_{\alpha}+ \\
& \quad \delta_{\mathrm{n}}(\mathrm{u}, \mathrm{y})_{\alpha} \beta \mathrm{t}_{\mathrm{n}-1}(\mathrm{z}) \beta\left[\mathrm{t}_{\mathrm{n}-1}(\mathrm{x}), \mathrm{t}_{\mathrm{n}-1}(\mathrm{y})\right]_{\alpha}=0
\end{aligned}
$$

Therefore, we get that

$\delta_{\mathrm{n}}(\mathrm{x}, \mathrm{y})_{\alpha} \beta \mathrm{t}_{\mathrm{n}-1}(\mathrm{z}) \beta\left[\mathrm{t}_{\mathrm{n}-1}(\mathrm{u}), \mathrm{t}_{\mathrm{n}-1}(\mathrm{y})\right]_{\alpha} \beta \mathrm{t}_{\mathrm{n}-1}(\mathrm{z}) \beta \delta_{\mathrm{n}}(\mathrm{x}, \mathrm{y})_{\alpha}$

$\beta \mathrm{t}_{\mathrm{n}-1}(\mathrm{z}) \beta\left[\mathrm{t}_{\mathrm{n}-1}(\mathrm{u}), \mathrm{t}_{\mathrm{n}-1}(\mathrm{y})\right]_{\alpha}=0$

This implies that

$$
0=-\delta_{\mathrm{n}}(\mathrm{x}, \mathrm{y})_{\alpha} \beta \mathrm{t}_{\mathrm{n}-1}(\mathrm{z}) \beta\left[\mathrm{t}_{\mathrm{n}-1}(\mathrm{u}), \mathrm{t}_{\mathrm{n}-1}(\mathrm{y})\right]_{\alpha} \beta \mathrm{t}_{\mathrm{n}-1}(\mathrm{z}) \beta \delta_{\mathrm{n}}(\mathrm{u}, \mathrm{y})_{\alpha} \beta \mathrm{t}_{\mathrm{n}-1}(\mathrm{z}) \beta
$$


$1 \quad\left[\mathrm{t}_{\mathrm{n}-1}(\mathrm{x}), \mathrm{t}_{\mathrm{n}-1}(\mathrm{y})\right]_{\alpha}$

Since $M$ is a prime $\Gamma$-ring, we have that

$\delta_{\mathrm{n}}(\mathrm{x}, \mathrm{y})_{\alpha} \beta \mathrm{t}_{\mathrm{n}-1}(\mathrm{z}) \beta\left[\mathrm{t}_{\mathrm{n}-1}(\mathrm{u}), \mathrm{t}_{\mathrm{n}-1}(\mathrm{y})\right]_{\alpha}=0$

The substitution of $(y+v)$ for $y$ in Lemma (2.9) ( i ), gives that

$\delta_{\mathrm{n}}(\mathrm{x}, \mathrm{y}+\mathrm{v})_{\alpha} \beta \mathrm{t}_{\mathrm{n}-1}(\mathrm{z}) \beta\left[\mathrm{t}_{\mathrm{n}-1}(\mathrm{x}), \mathrm{t}_{\mathrm{n}-1}(\mathrm{y}+\mathrm{v})\right]_{\alpha}=0$

$\delta_{\mathrm{n}}(\mathrm{x}, \mathrm{y})_{\alpha} \beta \mathrm{t}_{\mathrm{n}-1}(\mathrm{z}) \beta\left[\mathrm{t}_{\mathrm{n}-1}(\mathrm{x}), \mathrm{t}_{\mathrm{n}-1}(\mathrm{y})\right]_{\alpha}+$

$\delta_{\mathrm{n}}(\mathrm{x}, \mathrm{y})_{\alpha} \beta \mathrm{t}_{\mathrm{n}-1}(\mathrm{z}) \beta\left[\mathrm{t}_{\mathrm{n}-1}(\mathrm{x}), \mathrm{t}_{\mathrm{n}-1}(\mathrm{v})\right]_{\alpha}+$

$\delta_{\mathrm{n}}(\mathrm{x}, \mathrm{v})_{\alpha} \beta \mathrm{t}_{\mathrm{n}-1}(\mathrm{z}) \beta\left[\mathrm{t}_{\mathrm{n}-1}(\mathrm{x}), \mathrm{t}_{\mathrm{n}-1}(\mathrm{y})\right]_{\alpha}+$

$\delta_{\mathrm{n}}(\mathrm{x}, \mathrm{v}){ }_{\alpha} \beta \mathrm{t}_{\mathrm{n}-1}(\mathrm{z}) \beta\left[\mathrm{t}_{\mathrm{n}-1}(\mathrm{x}), \mathrm{t}_{\mathrm{n}-1}(\mathrm{v})\right]_{\alpha}=0$

By utilizing Lemma ( 2.9) ( i ), we obtain that

$\delta_{\mathrm{n}}(\mathrm{x}, \mathrm{y})_{\alpha} \beta \mathrm{t}_{\mathrm{n}-1}(\mathrm{z}) \beta\left[\mathrm{t}_{\mathrm{n}-1}(\mathrm{x}), \mathrm{t}_{\mathrm{n}-1}(\mathrm{v})\right]_{\alpha}+$

$\delta_{\mathrm{n}}(\mathrm{x}, \mathrm{y})_{\alpha} \beta \mathrm{t}_{\mathrm{n}-1}(\mathrm{z}) \beta\left[\mathrm{t}_{\mathrm{n}-1}(\mathrm{x}), \mathrm{t}_{\mathrm{n}-1}(\mathrm{y})\right]_{\alpha}=0$

Consequently, we have

$\delta_{\mathrm{n}}(\mathrm{x}, \mathrm{y})_{\alpha} \beta \mathrm{t}_{\mathrm{n}-1}(\mathrm{z}) \beta\left[\mathrm{t}_{\mathrm{n}-1}(\mathrm{x}), \mathrm{t}_{\mathrm{n}-1}(\mathrm{v})\right]_{\alpha} \beta \mathrm{t}_{\mathrm{n}-1}(\mathrm{z}) \beta \delta_{\mathrm{n}}(\mathrm{x}, \mathrm{y})_{\alpha}$

$\beta \mathrm{t}_{\mathrm{n}-1}(\mathrm{z}) \beta\left[\mathrm{t}_{\mathrm{n}-1}(\mathrm{x}), \mathrm{t}_{\mathrm{n}-1}(\mathrm{v})\right]_{\alpha}=0$

This implies that

$0=\delta-{ }_{n}(\mathrm{x}, \mathrm{y})_{\alpha} \beta \mathrm{t}_{\mathrm{n}-1}(\mathrm{z}) \beta\left[\mathrm{t}_{\mathrm{n}-1}(\mathrm{x}), \mathrm{t}_{\mathrm{n}-1}(\mathrm{v})\right]_{\alpha} \beta \mathrm{t}_{\mathrm{n}-1}(\mathrm{z}) \beta \delta_{\mathrm{n}}(\mathrm{x}, \mathrm{v})_{\alpha}$

$\beta \mathrm{t}_{\mathrm{n}-1}(\mathrm{z}) \beta\left[\mathrm{t}_{\mathrm{n}-1}(\mathrm{x}), \mathrm{t}_{\mathrm{n}-1}(\mathrm{y})\right]_{\alpha}$

The fact that $\mathrm{M}$ is a prime number yields that

$\delta_{\mathrm{n}}(\mathrm{x}, \mathrm{y})_{\alpha} \beta \mathrm{t}_{\mathrm{n}-1}(\mathrm{z}) \beta\left[\mathrm{t}_{\mathrm{n}-1}(\mathrm{x}), \mathrm{t}_{\mathrm{n}-1}(\mathrm{v})\right]_{\alpha}=0$

Now, $\delta_{\mathrm{n}}(\mathrm{x}, \mathrm{y})_{\alpha} \beta \mathrm{t}_{\mathrm{n}-1}(\mathrm{z}) \beta\left[\mathrm{t}_{\mathrm{n}-1}(\mathrm{x}+\mathrm{u}), \mathrm{t}_{\mathrm{n}-1}(\mathrm{y}+\mathrm{v})\right]_{\alpha}=0$

$\delta_{\mathrm{n}}(\mathrm{x}, \mathrm{y})_{\alpha} \beta \mathrm{t}_{\mathrm{n}-1}(\mathrm{z}) \beta\left[\mathrm{t}_{\mathrm{n}-1}(\mathrm{x}), \mathrm{t}_{\mathrm{n}-1}(\mathrm{y})\right]_{\alpha}+$

$\delta_{\mathrm{n}}(\mathrm{x}, \mathrm{y})_{\alpha} \beta \mathrm{t}_{\mathrm{n}-1}(\mathrm{z}) \beta\left[\mathrm{t}_{\mathrm{n}-1}(\mathrm{x}), \mathrm{t}_{\mathrm{n}-1}(\mathrm{v})\right]_{\alpha}+$

$\delta_{\mathrm{n}}(\mathrm{x}, \mathrm{y})_{\alpha} \beta \mathrm{t}_{\mathrm{n}-1}(\mathrm{z}) \beta\left[\mathrm{t}_{\mathrm{n}-1}(\mathrm{u}), \mathrm{t}_{\mathrm{n}-1}(\mathrm{y})\right]_{\alpha}+$

$\delta_{\mathrm{n}}(\mathrm{x}, \mathrm{y})_{\alpha} \beta \mathrm{t}_{\mathrm{n}-1}(\mathrm{z}) \beta\left[\mathrm{t}_{\mathrm{n}-1}(\mathrm{u}), \mathrm{t}_{\mathrm{n}-1}(\mathrm{v})\right]_{\alpha}=0$

By employing equations ( 1 ), ( 2 ) and Lemma ( 2.9 ) (i ), we get

$\delta_{\mathrm{n}}(\mathrm{x}, \mathrm{y})_{\alpha} \beta \mathrm{t}_{\mathrm{n}-1}(\mathrm{z}) \beta\left[\mathrm{t}_{\mathrm{n}-1}(\mathrm{u}), \mathrm{t}_{\mathrm{n}-1}(\mathrm{v})\right]_{\alpha}=0$

(ii) We can obtain Lemma ( 2.10 ) ( ii ) by substituting $\beta$ in Lemma ( 2.10 ) (i ).

(iii) By substituting $\alpha+\beta$ for $\alpha$ in Lemma (2.10) (ii ), we have

$\delta_{\mathrm{n}}(\mathrm{x}, \mathrm{y})_{\alpha+\beta} \alpha \mathrm{t}_{\mathrm{n}-1}(\mathrm{z}) \alpha\left[\mathrm{t}_{\mathrm{n}-1}(\mathrm{u}), \mathrm{t}_{\mathrm{n}-1}(\mathrm{v})\right]_{\alpha+\beta}=0$

This implies that

$\delta_{\mathrm{n}}(\mathrm{x}, \mathrm{y})_{\alpha} \alpha \mathrm{t}_{\mathrm{n}-1}(\mathrm{z}) \alpha\left[\mathrm{t}_{\mathrm{n}-1}(\mathrm{u}), \mathrm{t}_{\mathrm{n}-1}(\mathrm{v})\right]_{\alpha}+$

$\delta_{\mathrm{n}}(\mathrm{x}, \mathrm{y})_{\alpha} \alpha \mathrm{t}_{\mathrm{n}-1}(\mathrm{z}) \alpha\left[\mathrm{t}_{\mathrm{n}-1}(\mathrm{u}), \mathrm{t}_{\mathrm{n}-1}(\mathrm{v})\right]_{\beta}+$

$\delta_{\mathrm{n}}(\mathrm{x}, \mathrm{y})_{\beta} \alpha \mathrm{t}_{\mathrm{n}-1}(\mathrm{z}) \alpha\left[\mathrm{t}_{\mathrm{n}-1}(\mathrm{u}), \mathrm{t}_{\mathrm{n}-1}(\mathrm{v})\right]_{\alpha}+$

$\delta_{\mathrm{n}}(\mathrm{x}, \mathrm{y}) \beta \alpha \mathrm{t}_{\mathrm{n}-1}(\mathrm{z}) \alpha\left[\mathrm{t}_{\mathrm{n}-1}(\mathrm{u}), \mathrm{t}_{\mathrm{n}-1}(\mathrm{v})\right]_{\beta}=0$

By applying equations ( i ) and ( ii ), we get that

$\delta_{\mathrm{n}}(\mathrm{x}, \mathrm{y})_{\alpha} \alpha \mathrm{t}_{\mathrm{n}-1}(\mathrm{z}) \alpha\left[\mathrm{t}_{\mathrm{n}-1}(\mathrm{u}), \mathrm{t}_{\mathrm{n}-1}(\mathrm{v})\right]_{\beta}+$

$\delta_{\mathrm{n}}(\mathrm{x}, \mathrm{y})_{\beta} \alpha \mathrm{t}_{\mathrm{n}-1}(\mathrm{z}) \alpha\left[\mathrm{t}_{\mathrm{n}-1}(\mathrm{u}), \mathrm{t}_{\mathrm{n}-1}(\mathrm{v})\right]_{\alpha}=0$ 
Therefore, we get that

$\delta_{\mathrm{n}}(\mathrm{x}, \mathrm{y})_{\alpha} \alpha \mathrm{t}_{\mathrm{n}-1}(\mathrm{z}) \alpha\left[\mathrm{t}_{\mathrm{n}-1}(\mathrm{u}), \mathrm{t}_{\mathrm{n}-1}(\mathrm{v})\right]_{\beta} \alpha \mathrm{t}_{\mathrm{n}-1}(\mathrm{z}) \alpha \delta_{\mathrm{n}}(\mathrm{x}, \mathrm{y})_{\alpha}$

$\alpha \mathrm{t}_{\mathrm{n}-1}(\mathrm{z}) \alpha\left[\mathrm{t}_{\mathrm{n}-1}(\mathrm{u}), \mathrm{t}_{\mathrm{n}-1}(\mathrm{v})\right]_{\beta}=0$

This yields that

$$
\begin{gathered}
0=-\delta_{\mathrm{n}}(\mathrm{x}, \mathrm{y})_{\alpha} \alpha \mathrm{t}_{\mathrm{n}-1}(\mathrm{z}) \alpha\left[\mathrm{t}_{\mathrm{n}-1}(\mathrm{u}), \mathrm{t}_{\mathrm{n}-1}(\mathrm{v})\right]_{\beta} \alpha \mathrm{t}_{\mathrm{n}-1}(\mathrm{z}) \alpha \\
\delta_{\mathrm{n}}(\mathrm{x}, \mathrm{y})_{\beta} \alpha \mathrm{t}_{\mathrm{n}-1}(\mathrm{z}) \alpha\left[\mathrm{t}_{\mathrm{n}-1}(\mathrm{u}), \mathrm{t}_{\mathrm{n}-1}(\mathrm{v})\right]_{\alpha}
\end{gathered}
$$

The fact that $\mathrm{M}$ is a prime $\Gamma$-ring gives that

$$
\delta_{\mathrm{n}}(\mathrm{x}, \mathrm{y})_{\alpha} \alpha \mathrm{t}_{\mathrm{n}-1}(\mathrm{z}) \alpha\left[\mathrm{t}_{\mathrm{n}-1}(\mathrm{u}), \mathrm{t}_{\mathrm{n}-1}(\mathrm{v})\right]_{\beta}=0
$$

\section{Theorem ( 2.11 )}

Every Jordan generalized higher reverse left (respectively right) centralizer of a 2-torsion free prime $\Gamma$-ring $\mathrm{M}$ into itself is a generalized higher reverse left (respectively right) centralizer of $M$.

\section{Proof:}

Let $\mathrm{T}=\left(\mathrm{T}_{\mathrm{i}}\right)_{\mathrm{i} \in \mathrm{N}}$ be a Jordan generalized higher reverse left (respectively right) centralizer of a prime $\Gamma$-ring $\mathrm{M}$ into itself. Since $M$ is a prime $\Gamma$-ring, then by employing Lemma ( 2.10 ) ( i ), we have that either $\delta_{\mathrm{n}}(\mathrm{x}, \mathrm{y})_{\alpha}=0$ or $\left[\mathrm{t}_{\mathrm{n}-1}(\mathrm{u}), \mathrm{t}_{\mathrm{n}-1}(\mathrm{v})\right]_{\alpha}=0$, for all $\mathrm{x}, \mathrm{y}, \mathrm{u}, \mathrm{v} \in \mathrm{M}$, $\alpha \in \Gamma$ and $\mathrm{n} \in \mathrm{N}$.

If $\left[\mathrm{t}_{\mathrm{n}-1}(\mathrm{u}), \mathrm{t}_{\mathrm{n}-1}(\mathrm{v})\right]_{\alpha} \neq 0$, for all $\mathrm{u}, \mathrm{v} \in \mathrm{M}, \alpha \in \Gamma$, then $\delta_{\mathrm{n}}(\mathrm{x}, \mathrm{y})_{\alpha}=0$, for all $\mathrm{x}, \mathrm{y} \in \mathrm{M}$ and $\mathrm{n} \in \mathrm{N}$. Hence, using Remark (2.8), we obtain that $\mathrm{T}$ is a generalized higher reverse left (respectively right) centralizer of $\mathrm{M}$. If $\left[\mathrm{t}_{\mathrm{n}-1}(\mathrm{u}), \mathrm{t}_{\mathrm{n}-1}(\mathrm{v})\right]_{\alpha}=0$, for all $\mathrm{u}, \mathrm{v} \in \mathrm{M}$ and $\mathrm{n} \in \mathrm{N}$, then $\mathrm{M}$ is commutative.

By utilizing Lemma (2.5) ( i ), we have that

$$
\begin{aligned}
\mathrm{T}_{\mathrm{n}}(\mathrm{x} \alpha \mathrm{y}+\mathrm{x} \alpha \mathrm{y}) & =\mathrm{T}_{\mathrm{n}}(2 \mathrm{x} \alpha \mathrm{y}) \\
= & 2 \mathrm{~T}_{\mathrm{n}}(\mathrm{x} \alpha \mathrm{y}) \\
= & 2 \sum_{\mathrm{i}=1}^{\mathrm{n}} \mathrm{T}_{\mathrm{i}}(\mathrm{y}) \alpha \mathrm{t}_{\mathrm{i}-1}(\mathrm{x})
\end{aligned}
$$

Since $\mathrm{M}$ is a 2-torsion free $\Gamma$-ring, we get that

$$
\mathrm{T}_{\mathrm{n}}(\mathrm{x} \alpha \mathrm{y})=\sum_{\mathrm{i}=1}^{\mathrm{n}} \mathrm{T}_{\mathrm{i}}(\mathrm{y}) \alpha \mathrm{t}_{\mathrm{i}-1}(\mathrm{x}) \text {. }
$$

Then $\mathrm{T}$ is a generalized higher reverse left (respectively right) centralizer of $\mathrm{M}$.

\section{Proposition ( 2.11 )}

Let $\mathrm{T}=\left(\mathrm{T}_{\mathrm{i}}\right)_{\mathrm{i} \in \mathrm{N}}$ be a Jordan generalized higher reverse left (respectively right) centralizer of a 2-torsion free $\Gamma$ ring $\mathrm{M}$ into itself, such that $\mathrm{x} \alpha \mathrm{y} \beta \mathrm{x}=\mathrm{x} \beta \mathrm{y} \alpha \mathrm{x}$, for all $\mathrm{x}, \mathrm{y} \in \mathrm{M}$ and $\alpha, \beta \in \Gamma$. Then $\mathrm{T}$ is a Jordan triple generalized higher reverse left (respectively right) centralizer of $\mathrm{M}$.

\section{Proof:}

The substitution of $b$ for ( $\mathrm{x} \alpha \mathrm{y}+\mathrm{y} \alpha \mathrm{x})$ in Lemma ( 2.5 ) ( $\mathrm{i}$ ), we have that 


$$
\begin{aligned}
& =\sum_{\mathrm{i}=1}^{\mathrm{n}} \mathrm{T}_{\mathrm{i}}(\mathrm{y}) \quad \beta \mathrm{t}_{\mathrm{i}-1}(\mathrm{x}) \alpha \mathrm{t}_{\mathrm{i}-1}(\mathrm{x})+\mathrm{T}_{\mathrm{i}}(\mathrm{x}) \quad \beta \mathrm{t}_{\mathrm{i}-1}(\mathrm{y}) \alpha \mathrm{t}_{\mathrm{i}-1}(\mathrm{x})+ \\
& \mathrm{T}_{\mathrm{i}}(\mathrm{x}) \alpha \mathrm{t}_{\mathrm{i}-1}(\mathrm{y}) \quad \beta \mathrm{t}_{\mathrm{i}-1}(\mathrm{x})+\mathrm{T}_{\mathrm{i}}(\mathrm{x}) \alpha \mathrm{t}_{\mathrm{i}-1}(\mathrm{x}) \quad \beta \mathrm{t}_{\mathrm{i}-1}(\mathrm{y}) \ldots(1)
\end{aligned}
$$

Moreover, we get that

$T_{n}(x \alpha(x \beta y+y \beta x)+(x \beta y+y \beta x) \alpha x)$

$=\mathrm{t}_{\mathrm{n}}(\mathrm{x} \alpha \mathrm{x} \beta \mathrm{y}+\mathrm{x} \alpha \mathrm{y} \beta \mathrm{x}+\mathrm{x} \beta \mathrm{y} \alpha \mathrm{x}+\mathrm{y} \beta \mathrm{x} \alpha \mathrm{x})$

$=T_{n}(y \beta x \alpha)+T_{n}(x \alpha x \beta y)+T_{n}(x \alpha y \beta x+x \beta y \alpha x)$

$=\sum_{\mathrm{i}=1}^{\mathrm{n}} \mathrm{T}_{\mathrm{i}}(\mathrm{y}) \boldsymbol{\beta} \mathrm{t}_{\mathrm{i}-1}(\mathrm{x}) \alpha \mathrm{t}_{\mathrm{i}-1}(\mathrm{x})+\mathrm{T}_{\mathrm{i}}(\mathrm{x}) \alpha \mathrm{t}_{\mathrm{i}-1}(\mathrm{x}) \boldsymbol{\beta} \mathrm{t}_{\mathrm{i}-1}(\mathrm{y})+$

$\mathrm{T}_{\mathrm{n}}(\mathrm{x} \alpha \mathrm{y} \beta \mathrm{x}+\mathrm{x} \beta \mathrm{y} \alpha \mathrm{x}) \ldots(2)$

By comparing equations ( 1 ), ( 2 ) and the fact that $\mathrm{x} \alpha \mathrm{y} \beta \mathrm{x}=\mathrm{x} \beta \mathrm{y} \alpha \mathrm{x}$,

for all $\mathrm{x}, \mathrm{y} \in \mathrm{M}$ and $\alpha, \beta \in \Gamma$, we have that

$\mathrm{T}_{\mathrm{n}}(\mathrm{x} \alpha \mathrm{y} \beta \mathrm{x}+\mathrm{x} \alpha \mathrm{y} \beta \mathrm{x})=2 \mathrm{~T}_{\mathrm{n}}(\mathrm{x} \alpha \mathrm{y} \beta \mathrm{x})$

$=2 \sum_{\mathrm{i}=1}^{\mathrm{n}} \mathrm{T}_{\mathrm{i}}(\mathrm{x}) \boldsymbol{\beta} \mathrm{t}_{\mathrm{i}-1}(\mathrm{y}) \alpha \mathrm{t}_{\mathrm{i}-1}(\mathrm{x})$

Since $\mathrm{M}$ is a 2-torsion free $\Gamma$-ring, we obtain that $\mathrm{F}$ is a Jordan triple generalized higher reverse left (respectively right) centralizer of $\mathrm{M}$.

\section{References:}

[1] W. E Barnes." On The Г-Rings of Nobusawa ", Pac. J. Math , .18, (3) , 411-422, 1966.

[2] Y . Ceven . and M . A Ozturk , " On Jordan Generalized Derivations in Gamma Rings ", Hacet. J. Math Stat., 33,.11-14, 2004.

[3] B.M Hamad, " (U,M) derivations on $\Gamma$-Rings ", M.Sc.Thesis, Department of mathematics, College of Education, Al-Mustansiriya University, 2012

[4] M.F Hoque and A.C Paul, " On Centralizers of Semiprime Gamma Rings ", International Mathematical Forum, 6(13), 627- 638, 2011.

[5] S . Kyuno , " On prime gamma Rings ", Pac. J. Math, 75(1), 185 - 190, 1978.

[6] S.M Salih, " generalized higher left Centralizer of prime Rings ", Int.J. curr.Res. , 8( 11), 40966 40975, 2016.

[7] F.R Jarullah and S.M Salih , " Higher Reverse Left (respectively Right) Centralizer of Prime $\Gamma$ Rings ", to appear .

[8] F.R Jarullah and S.M Salih , " Jordan Higher Reverse Left (respectively Right) Centralizer of Prime Rings ", to appear .

[9] F.R Jarullah and S.M Salih , " Jordan Generalized Higher Reverse Left (respectively Right) Centralizer of Prime Rings ", to appear . 